\title{
Altruism in a Time of Crisis: Dissociable Social Valuation and Perception during COVID-19 in the United States
}

Yi Luo

Virginia Tech https://orcid.org/0000-0002-6169-8938

\section{Anastasia Shuster}

Icahn School of Medicine at Mount Sinai https://orcid.org/0000-0002-5455-424X

Dongil Chung

Ulsan National Institute of Science and Technology https://orcid.org/0000-0003-1999-0326

\section{Madeline O'Brien}

Icahn School of Medicine at Mount Sinai

Matthew Heflin

Icahn School of Medicine at Mount Sinai

Ofer Perl

Icahn School of Medicine at Mount Sinai

Kaustubh Kulkarni

Icahn School of Medicine at Mount Sinai

\section{Soojung $\mathrm{Na}$}

Icahn School of Medicine at Mount Sinai

Vincenzo Fiore

Icahn School of Medicine at Mount Sinai

Read Montague

Virginia Tech Carilion Research Institute

Xiaosi Gu ( $\nabla$ xiaosi.gu@mssm.edu )

Icahn School of Medicine at Mount Sinai

\section{Article}

Keywords: COVID-19, human behavior, altruism, self-prioritization

Posted Date: September 1st, 2021

DOI: https://doi.org/10.21203/rs.3.rs-820359/v1 
License: (c) (i) This work is licensed under a Creative Commons Attribution 4.0 International License. Read Full License 


\section{Altruism in a Time of Crisis: Dissociable Social Valuation and Perception during COVID- 19 in the United States}

Yi Luo ${ }^{1}$, Anastasia Shuster ${ }^{2}$, Dongil Chung ${ }^{3}$, Madeline O’Brien ${ }^{4}$, Matt Heflin², Ofer Perl², Kaustubh Kulkarni ${ }^{4}$, Soojung $\mathrm{Na}^{4}$, Vincenzo G. Fiore ${ }^{2}$, P. Read Montague ${ }^{1,5,6,7}$, Xiaosi Gu ${ }^{2,4^{*}}$

1 Fralin Biomedical Research Institute at VTC, Virginia Tech, Roanoke, VA, USA, 24016

2 Department of Psychiatry, Icahn School of Medicine at Mount Sinai, New York, NY, USA, 10029

3 Department of Biomedical Engineering, Ulsan National Institute of Science and Technology, Ulsan, South Korea

4 Nash Family Department of Neuroscience, Icahn School of Medicine at Mount Sinai, New York, NY, USA, 10029

5 Wellcome Trust Centre for Neuroimaging, University College London, London, UK

6 Department of Physics, Virginia Tech, Blacksburg, VA, USA, 24061

7 Virginia Tech-Wake Forest School of Biomedical Engineering and Mechanics, Blacksburg, VA, USA, 24061

*Correspondence author: Xiaosi Gu, PhD (xiaosi.gu@mssm.edu) 


\begin{abstract}
Human prosocial behaviors are constantly shaped by the push-and-pull between societal need for cooperation and one's natural tendency to self-prioritize. Nevertheless, it remains elusive how our valuation and perceptual systems might contribute to altruistic acts under the influence of a real-world crisis. Here, using computational modeling and a game-theoretic approach, we investigated how the coronavirus pandemic perturbed altruistic choices in the United States, April-May, 2020. Overall, people made more altruistic choices as the pandemic worsened, an effect primarily driven by increased preference for social welfare. Paradoxically, participants also processed self-relevant information (i.e., "self-prioritization") more efficiently at the perceptual level, as the pandemic became worse. These effects were not observed one year later (May-June, 2021) when the variability of the pandemic diminished. Furthermore, individuals' prosocial choices and preferences did not correlate with their self-prioritization efficiency. Collectively, these results revealed a more nuanced view of human altruism - that as a dynamic and context-dependent construct, altruism can co-exist with increased attention to the self.
\end{abstract}




\section{Introduction}

Prosocial behaviors are critical for the evolution of human cooperation and civilization ${ }^{1,2}$. Accumulating data suggest that as a social species, humans have a strong inclination to behave altruistically, improving others' welfare often at a cost to their own welfare ${ }^{3-5}$. These acts are considered critical for maintaining group norms and survival in the long term ${ }^{5,6}$ and are heavily shaped by the formation of human society. Despite its ubiquity, altruism is not a static construct. Instead, the extent to which humans exhibit altruistic behaviors can change dynamically and rapidly based on the context, as shown in previous laboratory studies ${ }^{7,8}$. For example, altruism changes as a function of recipient identity - people donate more money to recipients with certain characteristics, such as those they believe to be "deserving" (i.e., an established charity) than others (e.g., an anonymous individual) ${ }^{9}$. Moreover, people make less selfish choices and exhibit increased trustworthiness when they are under high stress environment (e.g., time or social pressures) ${ }^{10,11}$. These experimental data pinpoint a context-dependent aspect of human altruism that is still not well understood at a mechanistic level.

Crises are an extreme situation where prosocial behavior plays a pivotal role in ensuring cooperation and survival. Such adverse events are also known to elicit biologically rooted "fightor-flight" responses ${ }^{12}$ which are encoded by brain structures preserved across species, supporting the automaticity and evolutionary nature of these self-prioritized perception and responses ${ }^{13,14}$. In contrast, behaviors such as self-initiated rescue of stranded others and donation to those who lost their homes are widely observed following disasters like 9/11 or Hurricane Katrina, indicating that people do care about others' wellbeing ${ }^{15}$. The push-and-pull between our perceptual and valuation systems make it debatable whether a crisis automatically makes people more selfish or more prosocial and how these two systems might interact to contribute to prosocial behavior ${ }^{16}$.

Thus far, much of the empirical work studying altruism was done in experimental settings with artificial manipulations of the context (e.g., stress), often sampling college students ${ }^{17,18}$. It remains elusive how real-world events that take place outside the lab could impact altruistic behaviors in large diverse samples. The answer to this question is fundamental to understanding the role of altruism in coping with crises. Here, using the COVID-19 pandemic as a natural stressor that caused a significant perturbation to social cognition and behavior, we examined the dynamics of human altruism at both valuation and perceptual levels between April $2^{\text {nd }}, 2020$ and June $4^{\text {th }}, 2020$ (Fig 1A, B) - approximately spanning the first wave of the COVID-19 pandemic in the United States. A total of 1,512 current U.S. residents enrolled in our study on April $2^{\text {nd }}$, 2020 (T1), and completed decision-making tasks every 14 days (i.e., at time points T1, T3, T5, T7, and T9; Fig. 1B) and self-reports every seven days (i.e., at all time points from T1 to T10; Fig. S1). A control study was conducted a year later (May - June 2021) as a comparison to the main study (see Methods for details).

Specifically, each participant was asked to play both a modified version of dictator game (DG) ${ }^{19}$, a paradigm that has been extensively used to probe altruistic choices (Fig. 1C); and a perceptual task (self-tagging task; Fig. 1D) which quantifies how people perceptually prioritize self-relevant information over information pertaining others (i.e., self-prioritization) ${ }^{20}$. Using computational modeling, we found that after controlling for the effects of time and demographic characteristics, altruistic choices - primarily driven by a greater preference for social welfare - 
increased as a function of the worsening of the pandemic over 9 weeks, indexed by a 7-day change in new cases. Paradoxically, people also prioritized self-relevant information at the perceptual level as the pandemic became worse. Nevertheless, such perceptual effect did not correlate with altruistic preferences or choices at any time point. Taken together, our results suggest that human altruism is a dynamic construct that can be amplified by the worsening of a naturalistic stressor (i.e., pandemic); and that humans' natural tendency to self-prioritize at the perceptual level does not necessarily interfere with our ability to make altruistic choices during a time of extreme crisis.

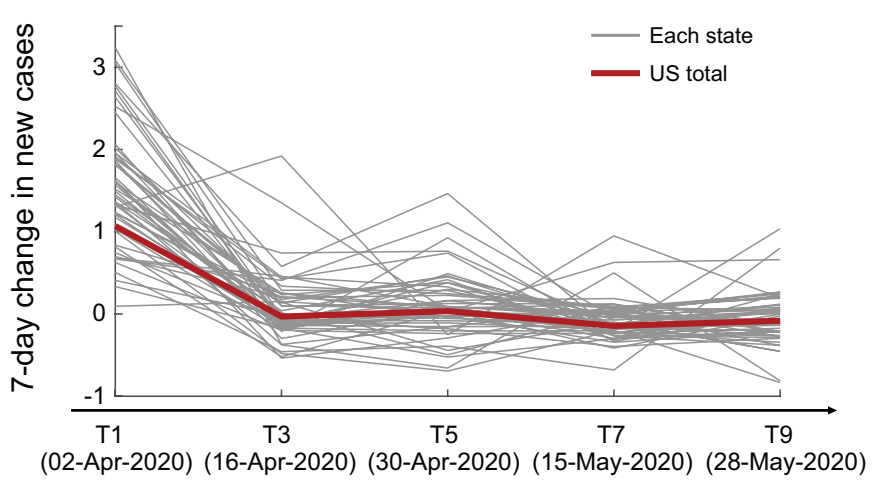

B

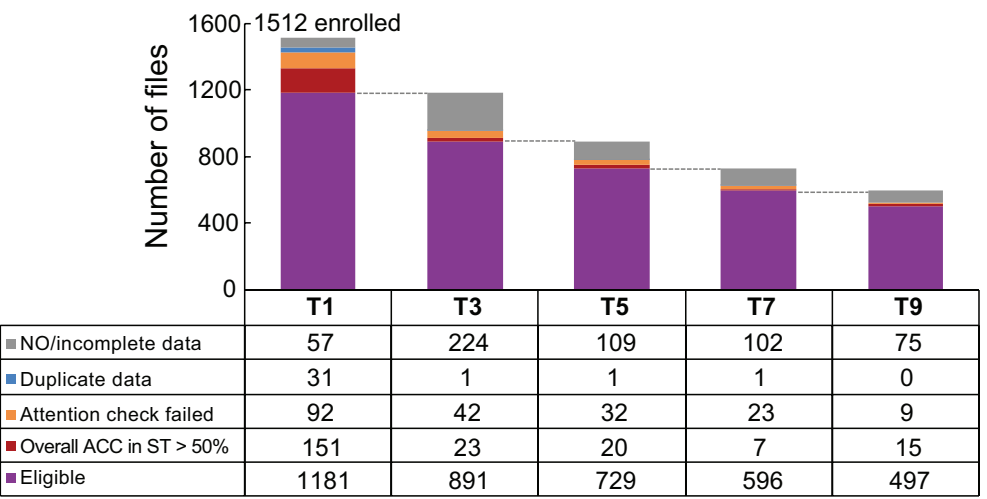

C

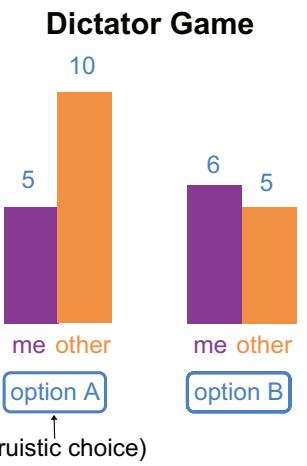

D
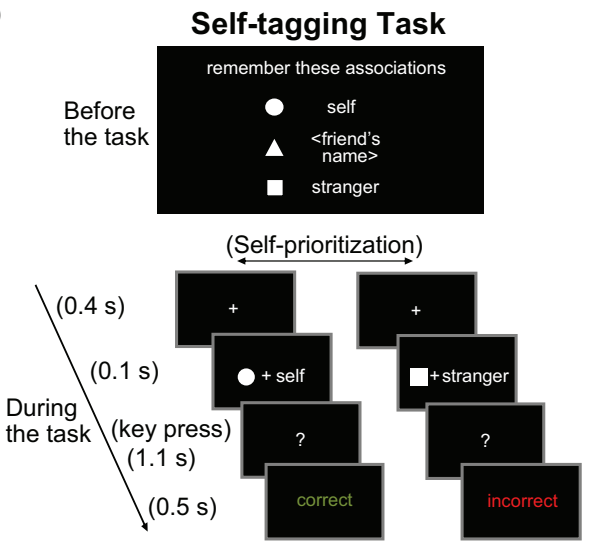

Figure 1. Data acquisition scheme and experiment design for the main study (2020 cohort). A. The 7-day change in new cases over behavior acquisition time points in each state (gray lines) and the USA average (red line) during April and May in 2020. The 7-day change was defined as the fractional change in new case number at one time point compared to new case number as of 7 days before. B. Data acquisition scheme, dropouts, and exclusions. ST, self-tagging task. C. An example trial of the dictator game. The monetary allocations in each option vary among trials. D. Examples of shape-label associations and two trials (one self-matched, the other stranger-matched) in the self-tagging task. Self-prioritization is quantified as the accuracy for self-matched pairs subtracted by the accuracy for stranger-matched pairs.

\section{Results}

A faster pace of the pandemic leads to increased altruistic choices 
First, we investigated the extent to which participants displayed altruistic behaviors towards anonymous partners in the DG (Fig. 1C) over 9 weeks. In each round, two options of monetary allocation between self and an anonymous partner were displayed. Participants were asked to make a series of choices ( 24 trials) between two options where one was an altruistic option and the other was a selfish option. Each participant's altruism level was defined as the probability of choosing the altruistic option across all trials. With a repeated-measures analysis of variance (ANOVA) test, we found a significant main effect of time, in that the percentage of altruistic choices generally decreased over time $\left(F(4,1984)=8.47, p<0.001, \eta_{p}^{2}=0.017\right.$; Fig. $\left.2 \mathrm{~A}\right)$. Post hoc analyses suggest that this effect was primarily contributed by a drop in altruistic levels from $\mathrm{T} 1$ to subsequent time points (all $p$ values $<0.05$ ).

Importantly, we asked how altruistic choices were affected by the severity of the pandemic. To do so, we calculated the 7-day change in new cases for each week, a standard index representing how fast the pandemic was worsening. We then fit a linear mixed-effects model which estimated how the 7-day change in new cases predicted altruistic choice after controlling for data acquisition time, sex, and age (see Methods for details). We again found that the probability of altruistic choice was positively associated with the 7-day change (unstandardized $\beta=0.013$, se = $0.004,95 \%$ confidence interval $[C I]=0.004-0.021, t(1754)=2.925, p=0.003$; Fig. $2 \mathrm{~B})$, over and above the effects of time, sex, and age (see details in Table S1). Such an association was not observed against the number of new cases itself (unstandardized $\beta=-8.36 \mathrm{E}-05$, se $=5.79 \mathrm{E}-05$, $95 \%$ CI, -1.97E-04 - 3.00E-05, $t(1433)=-1.445, p=0.149$; see details in Table S2). In other words, participants were more altruistic when there was a larger weekly increase in new cases in the week preceding the day of task completion. Taken together, these model-agnostic results demonstrate a positive impact of the crisis on increasing levels of altruism. 
MAIN STUDY (2020)

A
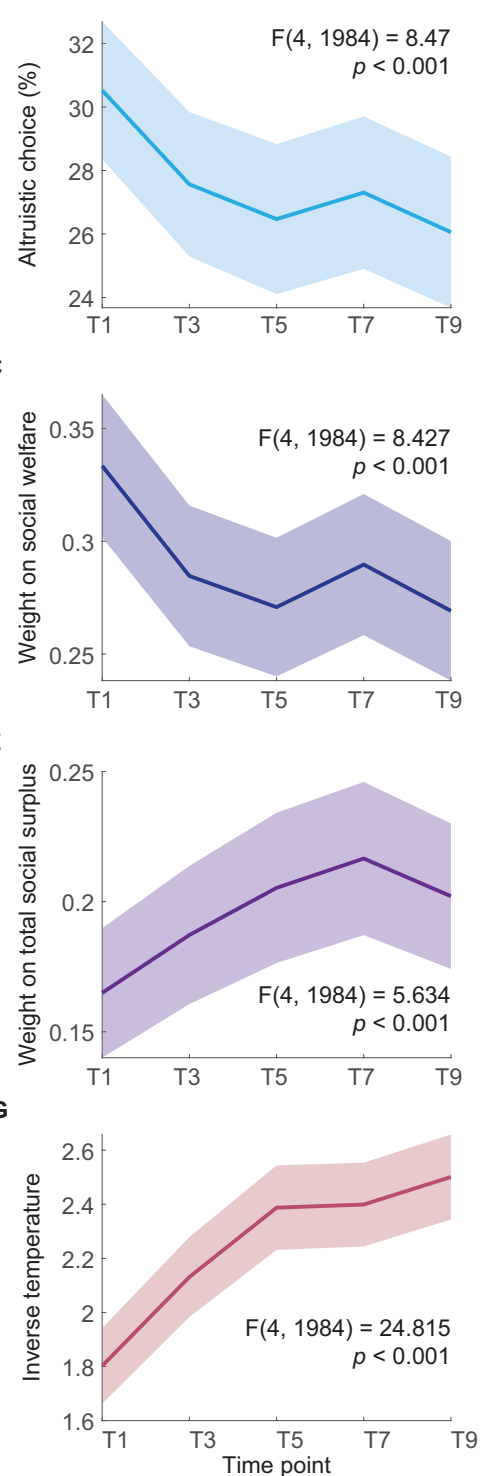

PANDEMIC

B

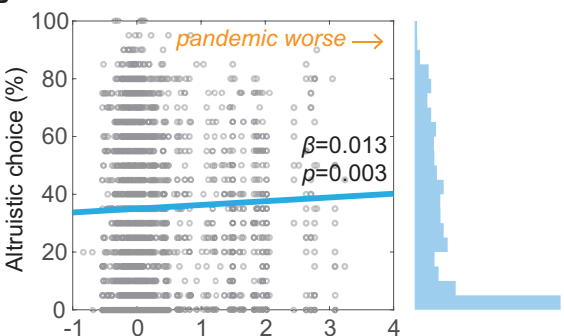

D

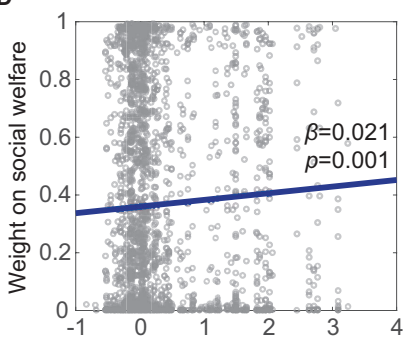

F
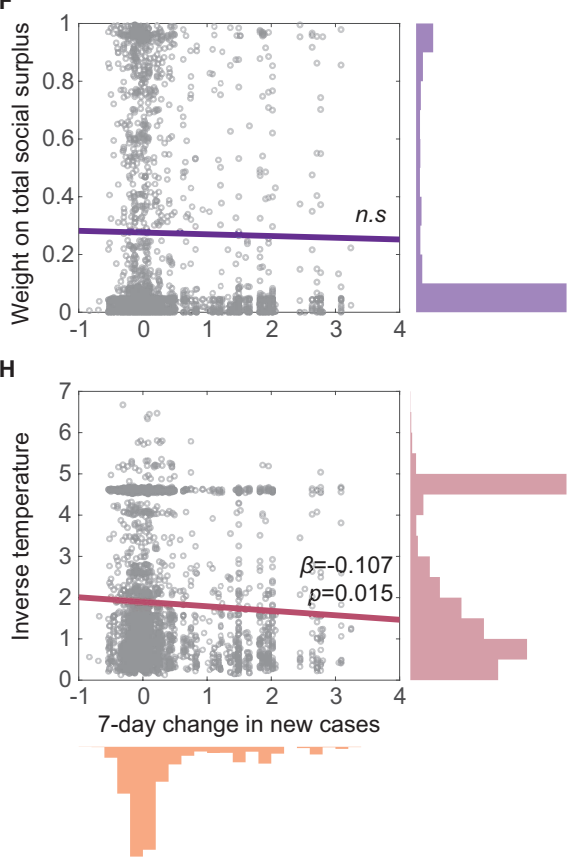

COMPARISON STUDY (2021)
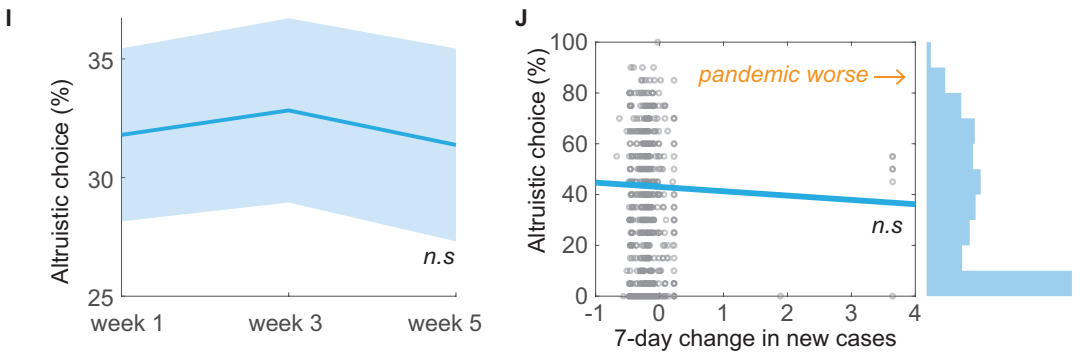
Figure 2. Altruism in the dictator game at each time point and its association with the 7-day change in new cases. A. Probability of altruistic choice at each time point in the 2020 cohort. B. The probability of altruistic choice against the 7-day change in new cases, pooling all time points in the 2020 cohort (Trend line: $y=0.013 x+0.343$ ). C. The model-based weight on social welfare at each time point in the 2020 cohort. D. The model-based weight on social welfare against the 7-day change in new cases, pooling all time points in the 2020 cohort (Trend line: $\mathrm{y}=0.021 \mathrm{x}+$ 0.359). E. The model-based weight on total social surplus at each time point in the 2020 cohort. F. The model-based weight on total social surplus against the 7-day change in new cases, pooling all time points in the 2020 cohort. (Trend line: $y=-0.006 x+0.277$ ). G. The inverse temperature at each time point in the 2020 cohort. $\mathbf{H}$. The inverse temperature against the 7-day change in new cases, pooling all time points in the 2020 cohort (Trend line: $y=-0.107$ $\mathrm{x}+1.940$ ). I. Probability of altruistic choice at each time point in the 2021 cohort. J. The probability of altruistic choice against the 7-day change in new cases, pooling all time points in the 2021 cohort (Trend line: $\mathrm{y}=-0.017 \mathrm{x}+$ 0.430). Trend lines were defined by the fixed effect obtained in mixed-model regression.

\section{Computational modeling reveals that the pandemic-modulated increase in altruistic choices was primarily driven by increased preference for social welfare}

To characterize the underlying motivations for altruistic choices, we constructed a computational model in which the utility of an option of monetary allocation was modulated by a social-welfare criterion ${ }^{21}$ (see Methods for details). Two parameters were estimated based on participants' choices in the DG at each time point; $\gamma$ denoting the extent to which one cares about maximizing the social welfare (i.e., an aggregate of their own payoffs and other's) over their own, and $\delta$ denoting the extent to which one weighs maximizing the total social surplus against helping the worse-off person (self included). With parameter recovery (see Methods for details), we confirmed that all parameters could be identified independently (weight on social welfare: $r=$ $0.94, p<0.001$; weight on total social surplus: $r=0.88, p<0.001$; inverse temperature $\tau: r=$ $0.87, p<0.001)$.

We found a significant main effect of time for the model-based weight on social welfare $(\gamma)$ $\left(F(4,1984)=8.427, p<0.001, \eta_{p}^{2}=0.017\right.$; Fig. $\left.2 C\right)$. Post hoc analyses suggest that this effect was primarily contributed by a drop in $\gamma$ from T1 to subsequent time points (all $p$ values $<0.05$ ). Consistent with the model-agnostic measure of altruism, after controlling for time, sex, and age, $\gamma$ was positively associated with the 7-day change in new cases (unstandardized $\beta=0.021$, se= $0.007,95 \% C I=0.008-0.034, t(1765)=3.193, p=0.001$; Fig. 2D), but not with the number of new cases per se (unstandardized $\beta=-1.66 \mathrm{E}-04$, se $=8.72 \mathrm{E}-05,95 \% C I=-3.37 \mathrm{E}-04-5.07 \mathrm{E}-06$, $t(1481)=-1.902, p=0.057)$, see details in Table S1 \& S2. In other words, people exhibited a stronger preference for social welfare when there was a higher weekly increase in new cases.

We found an opposite, yet significant, effect of time on the weight on total social surplus $(\delta)$ $\left(F(4,1948)=5.634, p<0.001, \eta_{p}^{2}=0.011\right.$; Fig. $\left.2 \mathrm{E}\right)$, with $\delta$ increasing from T1 to T5 ( $p<$ $0.029)$ and from T1 to T7 $(p=0.002)$. However, $\delta$ was not associated with the 7-day change in new cases (unstandardized $\beta=-0.006$, se $=0.006,95 \% C I=-0.018-0.007, t(1869)=-0.925, p$ $=0.355$; Fig. $2 \mathrm{~F}$ ), nor with new case number per se (unstandardized $\beta=8.15 \mathrm{E}-05$, se $=7.33 \mathrm{E}-$ $05,95 \% C I=-6.22 \mathrm{E}-05-2.25 \mathrm{E}-04, t(1099)=1.111, p=0.267)$, see details in Table S1 \& S2 . Taken together, these results suggest as the pandemic worsened, increased level of altruistic behavior was primarily driven by increased concern for social welfare.

We found a significant main effect of time for the model-based inverse temperature $(\tau)(F(4$, $1984)=24.815, p<0.001, \eta_{p}^{2}=0.048$; Fig. 2G). Post hoc analyses found that $\tau$ increased from T1 to T3 $(p<0.001)$, and continued to increase from T3 to T5 $(p=0.009)$. After controlling for 
time, sex, and age, $\tau$ was negatively associated with the 7-day change in new cases (unstandardized $\beta=-0.107$, se $=0.044,95 \% C I=-0.193--0.021, t(1918)=-2.437, p=0.015$; Fig. $2 \mathrm{H}$ ), but not with the number of new cases per se (unstandardized $\beta=0.001$, se $=4.67 \mathrm{E}-04$, $95 \% C I=-3.90 \mathrm{E}-05-0.002, t(1052)=1.876, p=0.061)$, see details in Table S1 \& S2. In other words, people's choices were more diffuse and variable when there was a higher weekly increase in new cases.

\section{People also show heightened attention to self at the perceptual level}

The aforementioned results suggest that as the pandemic became more pressing, individuals made more altruistic choices overall. However, it remains unclear how these explicit prosocial choices might be related to perceptual processing of self-relevant information. Self-prioritization - at the perceptual level - is known to promote adaptation to complex and dynamically changing environments, and optimizing self-survival ${ }^{20}$. Thus, one might expect that selfprioritization would be enhanced during a pandemic. To examine this hypothesis, each participant completed a self-tagging task in which they learned associations between three geometric shapes and three people (self, their best friend, and a stranger), and were asked to indicate whether a shape-label pairing was correct or not (Fig. 1D, see Methods for details). At the first time point (T1), participants were more accurate for self-matched pairs (i.e., when the shape was the one associated with "self", and correctly labeled) (mean \pm standard deviation, 87.9 $\pm 12.4 \%)$ than stranger-matched pairs $(75.1 \pm 17.3 \%$; paired-samples $t(496)=16.20, p<0.001)$. As in previous studies ${ }^{22}$, the difference in accuracy between self-matched and stranger-matched pairs was used as a measure of self-prioritization at each time point.

Similar to the dictator game, we found a significant main effect of time $(F(4,1984)=14.14, p<$ $\left.0.001, \eta_{p}^{2}=0.028\right)$. However, self-prioritization actually reduced from T1 to T3 $(p=0.014)$ and from T3 to T7 ( $p=0.038$ ) (Fig. 3A). Importantly, self-prioritization was positively predicted by the 7-day change in new cases (unstandardized $\beta=0.015$, se $=0.005,95 \% C I=0.006-0.025$, $t(2153)=3.099, p=0.002$; Fig. 3B) beyond the effects of time, sex, and age (see details in Table $\mathrm{S} 3$ ), but not by the number of new cases per se (unstandardized $\beta=-8.72 \mathrm{E}-06$, se $=4.49 \mathrm{E}-05$, $95 \% C I=-9.68 \mathrm{E}-05-7.93 \mathrm{E}-05, t(1063)=-0.194, p=0.846)$, see details in Table S3. In other words, participants became more efficient at percpetual processing of self-relevant stimuli when there was a larger weekly increase in new COVID-19 cases. We will examine how this perceptual effect directly relates to altruistic choices next.

\section{Altruistic choices and preferences were not predicted by self-prioritization at the perceptual level}

Thus far, we have observed that in spite of an increase in self-prioritization at the perceptual level, altruistic choices still became more frequent as the pandemic worsened. This raised the possibility that these two levels of processes might not be associated. To directly examine this possibility, we investigated whether self-prioritization in the self-tagging task was correlated with altruism in the DG (see Methods for details). Indeed, self-prioritization was not associated with the model-agnostic altruistic choices (unstandardized $\beta=-0.009$, se $=0.020,95 \% C I=$ $0.047-0.029, t(1928)=-0.467, p=0.641)$, the model-based parameter $\gamma$ representing concern for social welfare (unstandardized $\beta=-0.005$, se $=0.030,95 \% C I=-0.063-0.053, t(1971)=$ $0.174, p=0.862$ ), the weight on social surplus $\delta$ (unstandardized $\beta=-0.026$, se $=0.028,95 \% C I$ 
$=-0.081-0.029, t(2098)=-0.914, p=0.361$ ), nor the inverse temperature $\tau$ (unstandardized $\beta=$ 0.165 , se $=0.190,95 \% C I=-0.209-0.538, t(2234)=0.864, p=0.388)$, see details in Table S4. Further pair-wise correlations confirmed that self-prioritization was not correlated with any of the measures in the DG at any of the time points (all $p$ values $>0.05$; Fig. 3C \& 3D). The change in self-prioritization between adjacent time points was not predictive of the change in the altruistic choice either (unstandardized $\beta=-0.022$, se $=0.018,95 \% C I=-0.058-0.014, t(1988)$ $=-1.184, p=0.237$ ), the model-based parameter $\gamma$ (unstandardized $\beta=-0.039$, se $=0.028,95 \%$ $C I=-0.0951-0.017, t(1988)=-1.374, p=0.170)$, the model-based parameter $\delta$ (unstandardized $\beta=-0.027, \mathrm{se}=0.028,95 \% C I=-0.081-0.027, t(1988)=-0.972, p=0.331)$, nor the modelbased parameter $\tau$ (unstandardized $\beta=0.289$, se $=0.192,95 \% C I=-0.087-0.665, t(1988)=$ $1.508, p=0.132$ ), see details in Table S5. Again, we further calculated pair-wise correlations and confirmed that the change in self-prioritization was not correlated with change in any of the measures in the DG at any of the time points (all $p$ values $>0.05$ ). These results suggest an orthogonal, instead of co-dependent, relationship between social valuation and social perception under the influence of a crisis. 
MAIN STUDY (2020)
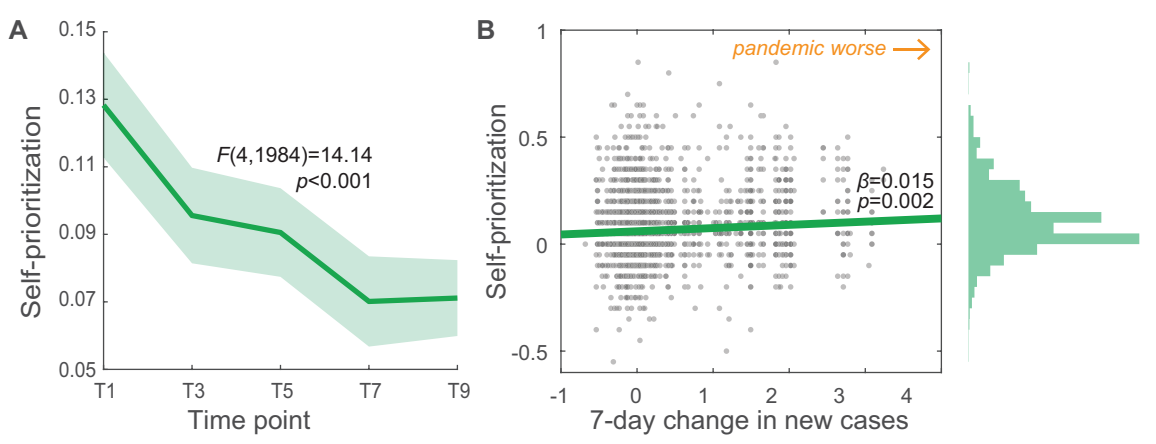

C

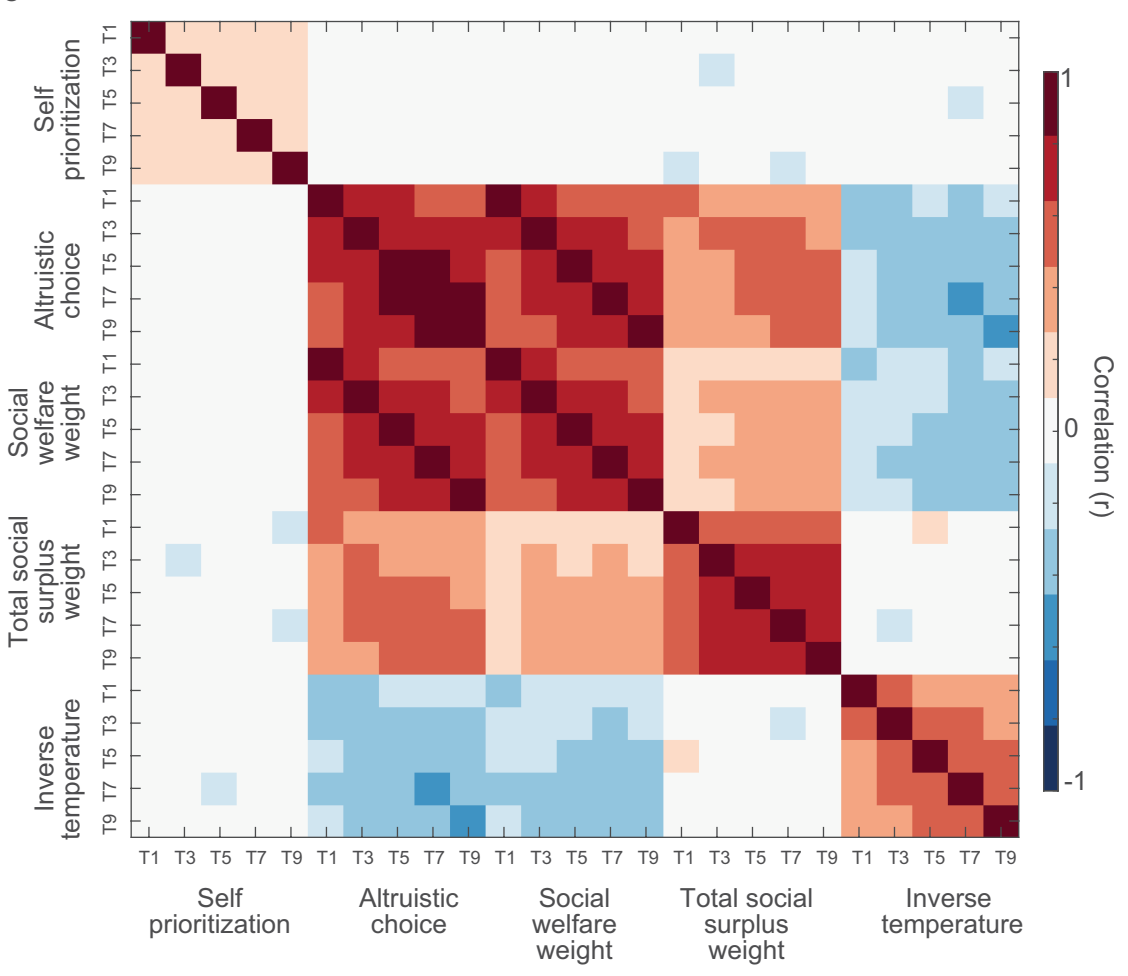

COMPARISON STUDY (2021)
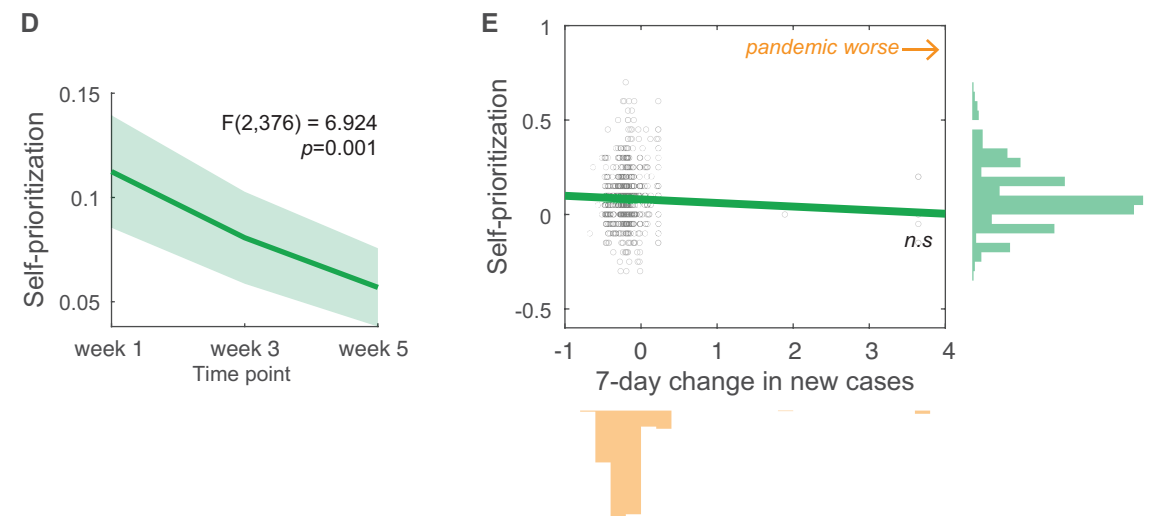
Figure 3. Self-prioritization in the self-tagging task at each time point and its association with the 7-day change in new cases and altruism. A. Self-prioritization at each time point in the 2020 cohort. B. Self-prioritization against the 7-day change in new cases, pooling all time points in the 2020 cohort. Trend line was defined by the fixed effect obtained in mixed-model regression $(\mathrm{y}=0.015 \mathrm{x}+0.063)$. C. Pearson correlation coefficients $(r)$ for pairs of selfprioritization in the self-tagging task and probability of altruistic choices as well as model-based parameters in the dictator game at each time point in the 2020 cohort. D. Self-prioritization at each time point in the 2021 cohort. E. Self-prioritization against the 7-day change in new cases, pooling all time points in the 2021 cohort (Trend line: $y=$ $-0.019 \mathrm{x}+0.084)$.

\section{1 cohort: no change in altruism over time.}

To further confirm that the observed effect so far was specific to the rapidly unfolding state of the emergency of the pandemic that was characteristic to the spring of 2020, we collected data from a new cohort from May $7^{\text {th }}, 2021$ to June $4^{\text {th }} 2021$, during a time when the 7-day change in severity had a very different, less variable, pattern from the 2020 cohort (Fig. S2A). A total of 360 current U.S. residents enrolled in this new cohort on May $7^{\text {th }}, 2021$ (week 1) and 189 of them completed both DG and the self-tagging task every 14 days (i.e., at time points week 1 , week 3, and week 5; Fig. S2B) and passed all data quality checks (see Methods for details).

In the 2021 cohort, the change in new cases was relatively flat compared to that of the 2020 cohort (Fig. S2A). The pattern of change in altruism was also flat - there was not a significant change over time in the percentage of altruistic choices $\left(F(2,376)=0.833, p=0.436, \eta_{p}^{2}=\right.$ 0.004; Fig. 2I). The main effect of time for the model-based weight on social welfare $(\gamma)$ was not significant either $\left(F\left(2,376=2.159, p=0.117, \eta_{p}^{2}=0.011\right.\right.$; Fig. S3A). These results in the new cohort support that the pandemic-modulated increase in altruistic choices, which was primarily driven by increased preference for social welfare, was specific to the first wave of the pandemic.

In addition, we found similar effects of time on the weight on total social surplus and inverse temperature in the 2021 cohort as those in the 2020 cohort. Specifically, time had a significant impact on the weight on total social surplus $(\delta)$ in the 2021 cohort $(F(2,376)=3.233, p=0.042$, $\eta_{p}^{2}=0.017$; Fig. S3C), with $\delta$ having a trend of increase from week1 to week5 $(p=0.057)$. The effect of time on the model-based inverse temperature $(\tau)$ was also significant in the 2021 cohort $\left(F(2,376)=13.077, p<0.001, \eta_{p}^{2}=0.065\right.$; Fig. 3E), with $\tau$ increasing from week1 to week $5(p$ $<0.001)$, as well as from week3 to week5 $(p=0.006)$.

We also tested the association between altruism and the pandemic severity in the 2021 cohort, with the effects of time, sex, and age controlled. No significant association was found between altruism and the 7-day change in new cases (Fig. 2J \& Fig. S3, see details in Table S6) or between altruism and the new case number per se (see details in Table S7). These findings also suggest that the pandemic-modulated increase in altruistic choices was specific to the first wave of the pandemic.

In the self-tagging task, similarly to the 2020 cohort, we found a significant main effect of time in the 2021 cohort $\left(F(2,376)=6.924, p=0.001, \eta_{p}^{2}=0.036\right)$. Self-prioritization reduced from week1 to week5 ( $p=0.001$ ) (Fig. 3D). However, self-prioritization was not associated with the 7-day change in new cases (unstandardized $\beta=-0.019$, se $=0.017,95 \% C I=-0.054-0.015$, $t(368.6)=-1.100, p=0.272$; Fig. $3 \mathrm{E}$ ) after controlling for the effects of time, sex, and age (see 
details in Table S8), nor with the number of new cases per se (unstandardized $\beta=1.06 \mathrm{E}-05$, se $=$ $1.89 \mathrm{E}-04,95 \% C I=-3.61 \mathrm{E}-04-3.82 \mathrm{E}-04, t(288.5)=0.056, p=0.955)$.

Lastly, consistent with our findings in the 2020 cohort, self-prioritization in the 2021 cohort was not associated with the model-agnostic altruistic choices (unstandardized $\beta=0.040$, se $=0.037$, $95 \% C I=-0.033-0.113, t(391.1)=1.079, p=0.281)$, the model-based parameter $\gamma$ representing concern for social welfare (unstandardized $\beta=0.101$, se $=0.057,95 \% C I=-0.012-0.214$, $t(412.2)=1.758, p=0.080$ ), the weight on social surplus $\delta$ (unstandardized $\beta=0.071$, se $=$ $0.061,95 \% C I=-0.048-0.190, t(442.4)=1.163, p=0.245)$, nor the inverse temperature $\tau$ (unstandardized $\beta=-0.266$, se $=0.360,95 \% C I=-0.973-0.440, t(460.2)=-0.739, p=0.460$; see details in Table S9). Further pair-wise correlations confirmed that self-prioritization was not correlated with any of the measures in the DG at any of the time points in the 2021 cohort (all $p$ values $>0.05)$. Again, we further calculated pair-wise correlations and confirmed that the change in self-prioritization was not correlated with change in any of the measures in the DG at any of the time points in the 2021 cohort (all $p$ values $>0.05$ ).

Overall, these results suggest that the main behavioral effects we observed during the first wave of the pandemic (spring 2020) were not found a year later (spring 2021), and that they were specifically tied to the initial worsening of the pandemic.

\section{Discussion}

While altruistic behaviors are common in human society, the effect of real-world crises on altruism has been long debated. Using the COVID-19 pandemic as a natural perturbation to human behavior, we demonstrated that participants made more choices that benefitted others more than themselves, as the pace of the pandemic became faster during the first wave of the pandemic; this effect was primarily driven by a heightened preference for social welfare over their own payoffs. We also found that individuals' altruistic choices and preferences were not associated with their perceptual efficiency related to the self. Our results indicate that altruism is a context-dependent construct that can be elevated by the acceleration of a real-world crisis, and that social valuation and perception are dissociable processes during complex social decisionmaking.

At the first time point in the main study (April 2 $2^{\text {nd }}, 2020$ ), around when the COVID-19 pandemic deteriorated most rapidly and nation-wide and stay-at-home orders were in effect in most states

${ }^{23}$, participants displayed the highest level of altruistic behaviors and preference for social welfare but the lowest weight on total social surplus in the DG. As time went by, and as the pandemic subsided in most states, people made less altruistic choices and had a decreased preference for social welfare but an increased weight on total social surplus. These results are consistent with previous findings in laboratory settings showing that altruism changes dynamically according to contextual manipulations such as stress ${ }^{17}$ and empathy induction ${ }^{18}$. Notably, after controlling for the impact of time and demographic characteristics, we found that people were still more altruistic and less selfish when there was a more severe deterioration of the COVID-19 crisis during the first wave, which also excludes the possibility that the changes in behavior were only driven by the simple repetition of the task. Further, our comparison study conducted in 2021 when the change in new cases was relatively flat found a flat pattern in the 
altruistic choice, which further confirmed that the increase in altruism was specifically associated with the initial worsening of the pandemic in 2020. This result echoes previous findings showing increased prosociality both during crises ${ }^{24,25}$ and in those who had experienced disaster and violence $^{26,27}$. In a similar vein, previous studies have revealed increased altruism in individuals who experienced past adverse life events, which was associated with a greater awareness of suffering, a heightened empathic concern, and a more positive orientation toward other people ${ }^{28-}$ 30 .

Our computational model took one step further by revealing that the increased altruism in the main study was primarily driven by an increased concern for social welfare during the first wave of the pandemic in 2020. Consistent with this finding, a recent study found that people displayed stronger intentions to avoid COVID-19 infection when the pandemic was framed as a public threat, which concerns the welfare of others, than when it was framed as a personal threat ${ }^{31}$. Indeed, social preference - sacrificing one's own benefit to increase the payoffs for all recipients - has been observed in a wide range of circumstances and has been associated with subjective reward ${ }^{32,33}$. Previous studies have found that people increase altruistic behaviors toward poor or needy recipients ${ }^{18,34}$. Potentially, as the COVID-19 pandemic rapidly unfolded and worsened, the distress and needs of others were more profound. Under such a crisis, people might have become more sensitive to the value of social welfare over one's own benefits, which predisposed them toward altruistic choices. On the other hand, other studies have shown that people are more likely to maximize social welfare and act more altruistically when interacting with in-group members ${ }^{35}$. Since social identity as a sense of membership in social groups is also contextdependent ${ }^{36}$, a rapidly worsening pandemic could form an emergent social identity in people framing them as members of a "pandemic society" with shared goals and outcomes during the crisis, which could elevate their concerns for social welfare over self-interest ${ }^{37}$.

At a perceptual level, humans prioritize information relevant to themselves, which may be an evolutionarily evolved instinct. The "fight-or-flight" response, for example, immediately increases the attention one pays to the self and improves adaptation to the environment when encountering uncertain or dangerous situations ${ }^{20}$. Although our study did not measure physiological responses directly, the finding of increased self-prioritization as the pandemic worsened is consistent with the notion that individuals tend to focus more on self-relevant information in endangering times. Indeed, perhaps the rapidly changing environment during the COVID-19 pandemic made people more self-focused for adaptive purposes. Interestingly, this pandemic-modulated self-prioritization effect showed an opposite pattern from altruistic behaviors and preferences which actually increased as the pandemic worsened more rapidly; the two sets of measures were also completely dissociable from each other at every time point. We speculate that perceptual responses are more closely associated with self-preservation during the crisis, whereas altruistic choices might be more complex and involve higher-level cognitive processes.

It should be noted that the DG in the current studies did not require participants to submit decisions within a limited time, so it is possible that early perceptual prioritization did play a role in information filtering and selection for altruistic decision-making but was attenuated by later cognitive processes. In line with this conjecture, a recent study had participants play the DG under high time pressure (1.5 seconds) and found that early attentional prioritization of the monetary outcomes for other over self (measured by the proportion of early gaze directed to 
payoffs of self or other) was predictive of their altruistic choices ${ }^{8}$. Furthermore, the authors showed that forcing individuals to attend to others' payoffs rather than their own increased altruistic choices under time pressure but not in free responses. This hints at the role of perceptual-level prioritization during the early stages of altruistic decision-making and the potential involvement of higher-level cognitive processes during the later stages. Here, our findings suggest the perceptual-level prioritization was dissociable from valuation when assessing the impact of a crisis. Still, the potential interaction/dissociation of perceptual- and higher-level cognitive processes as well as their underlying neural circuits need to be systematically examined with future empirical studies.

Some limitations of the current study should be noted. First, the contribution of local vs. nationwide COVID-19 severity cannot be fully disentangled. Our analyses were based on the severity of pandemic in each individual's residential state, since we consider the pandemic situation in one's own state was most salient to them. However, a person could be cognitively affected by the neighboring state(s), national-wide, or even the global COVID-19 severity, but our data will not reflect that. Second, our study quantified COVID-19 severity as well as its change based on daily new COVID-19 cases, which is a commonly reported indicator by the media in the United States. We should point out that the meaning of this indicator and how it reflects people's perception of COVID-19 severity has been changing, because the number of tests had increased dramatically since the first wave. Third, our study examined altruism based on allocations of money which is a general symbol of value, but the dynamic of crisis-specific altruistic decisionmaking, like donation of face masks and hand sanitizers in this case, is yet to be explored.

Taken together, our results demonstrate that a real-world crisis can both increase perceptual-level self-prioritization and decrease selfish behaviors at the same time. This finding provides a more nuanced view of prosocial behaviors under the threat of survival, and has far-reaching implications for the understanding of the origin and motivation of human cooperation and altruism.

\section{Materials and Methods}

\section{Participants}

Main study: the 2020 cohort. As displayed in Fig. 1A, 1512 participants (age (mean \pm SD), 35.1 \pm 13.1 years, range 18-77; 765 males, 736 females, 4 others, 7 unknowns because the subjects preferred not to say) enrolled in the online study on Prolific (www.prolific.co) on April $2^{\text {nd }}$, 2020. Survey data collection was conducted within a 24-hour time window, every 7 days between April 2 ${ }^{\text {nd }}, 2020$ and June $4^{\text {th }}, 2020$ (10 time points in total; Fig. S1), while task data collection was conducted within the same time window, every 14 days between the same time periods ( 5 time points in total; Fig. 1B). Exclusion criteria for data quality were: (1) voluntary dropping out of the study (567 or $37.5 \%$ of participants), (2) duplicate responses at any of the 5 time points for task data collection, resulting in removal from the study (34 or $2.2 \%$ of participants), (3) failing to respond accurately to attention-check questions (e.g., "If you are paying attention, please select 'most of the time"') at any task data acquisition time point (198 or $13.1 \%$ of participants)), and (4) having an accuracy below $50 \%$ in the self-tagging task at any time point (216 or $14.3 \%$ of participants). Overall, 497 participants (age, $35.4 \pm 13.0$ years, range 
18 - 76; 255 males, 240 females, 2 others; from 48 states) completed all surveys and both tasks at every task data acquisition time point without failing any data quality check at any of these time points. Behavioral responses for different subsets of participants with valid responses at all or some of the time points for task data collection are presented in Fig. S4. Consistent patterns of behaviors in both tasks indicate that our findings may have not been biased by the data exclusion procedure. Participants received base compensation for their time each week ( $\$ 7.25$ for weeks that included both surveys and behavioral tasks, $\$ 3$ for weeks that included only surveys), as well as a performance-based bonus. At week five, participants received an extra $\$ 10$ bonus for completing half of the study, and at week 10, participants received an extra $\$ 15$ bonus for completing the entire study.

Comparison study: the 2021 cohort. As displayed in Fig. S2, a new cohort of participants $(\mathrm{N}=$ 360 ; age, $33.8 \pm 10.6$ years, range 18-63; 177 males, 182 females, 1 unknown because the subject preferred not to say) enrolled in the comparison study on Prolific on May $7^{\text {th }}, 2021$. None of the participants in the 2020 cohort were included in the 2021 cohort. Data collection was conducted biweekly between May $7^{\text {th }}, 2021$ and June $4^{\text {th }}, 2021$ ( 3 time points in total). Exclusion criteria for data quality were the same as the 2020 cohort. Overall, 189 participants (age, $35.5 \pm$ 10.5 years, range 18 - 63; 92 males, 97 females; from 41 states) completed both tasks at every data acquisition time point without failing any data quality check at any of these time points. Behavioral responses for different subsets of participants with valid responses at all or some of the time points for task data collection are presented in Fig. S5. Again, consistent patterns of behaviors in both tasks indicate that our findings may have not been biased by the data exclusion procedure. Participants received base compensation for their time each week (\$4.35), as well as a performance-based bonus. At the last week, participants received an extra $\$ 10$ bonus for completing the entire study.

Informed consent was obtained from all participants via online consent forms. All experimental protocols were approved by the Institutional Review Board of the Icahn School of Medicine at Mount Sinai. All methods were performed in accordance with the relevant guidelines and regulations.

Analyses and results based on self-report surveys in the 2020 cohort in the main study are reported elsewhere as they are outside the scope of this study.

\section{COVID-19 severity}

Data for COVID-19 prevalence per state since the beginning of records in the US and until June $4^{\text {th }}, 2020$, as well as that from April $23^{\text {rd }}, 2021$ to June $4^{\text {th }}, 2021$ were obtained from New York Times (Sources: state and local health agencies. Population from Census Bureau.) ${ }^{38}$. For each state, the number of new COVID-19 cases at each time point in our experiment was defined as the average of daily new cases per million in the 7 days prior to that time point (Fig. S1). To characterize how fast the pandemic was worsening, we calculated the 7-day change in new cases for each state. Specifically, it was defined as the fractional change in new case number at one time point compared to new case number as of 7 days before (Fig. 1A for the 2021 cohort; Fig. S2A for the 2021 cohort). For instance, the 7-day change in new cases at T3 for the 2020 cohort was the difference between new case number at T3 and T2 (7 days before T3) scaled by new case number at $\mathrm{T} 2$. 


\section{Behavioral tasks}

All participants, at each task data acquisition time point (i.e., T1, T3, T5, T7, and T9 in the 2020 cohort; week1, week 3 , and week 5 in the 2021 cohort), were asked to complete the 24 rounds of DG ${ }^{19}$ (see details in Table S6), in which they chose between an altruistic option and a selfinterested option of monetary allocation between self and other (Fig. 1C). Four of the trials were catch trials for data quality control.

At each task data acquisition time point, participants were instructed before the self-tagging task 20 to learn associations between three geometric shapes and three people (self, their best friend, and a stranger). Then, in each trial of the self-tagging task, they were asked to indicate whether a shape-label pairing was correct (Fig. 1D). Each participant completed 107 trials in the task, composed of 20 self-matched pairs, 20 friend-matched pairs, 20 stranger-matched pairs, 10 selfunmatched pairs, 10 friend-unmatched pairs, 10 stranger-unmatched pairs, and 17 null trials in which a fixation cross was presented on the screen for 2.1 seconds.

\section{Data analysis}

Altruistic choices in the DG. The percentage of altruistic choices in non-catch trials in the DG was used as an indicator of altruism. We investigated the fluctuation of altruistic choice over time with a repeated measure (data acquisition time: T1/T3/T5/T7/T9) analysis of variance (ANOVA) test in the 2020 cohort, and another repeated measure (data acquisition time: week1/week3/week5) ANOVA test in the 2021 cohort.

Using linear mixed-effects regressions, in each cohort, we tested separately the effects of the 7day change in new cases and the number of new cases per se on altruistic choice over time, with slope and intercept included for random effects. We controlled for data acquisition time, sex, and age (altruistic choice $\sim 7$-day change in new cases + time + sex + age + (7-day change in new cases + time $\mid$ participant); altruistic choice $\sim$ new case number + time + sex + age + (new case number + time $\mid$ participant)).

Model-based analyses in the DG. As one of the models for social preference, the social-welfare model assumes that one prefers to increase the aggregate of their own payoffs and those of others (i.e., social welfare) during economic decision-making, caring especially about helping those with low payoffs ${ }^{21}$. The utility of an option of monetary allocation is discounted by socialwelfare criterion:

$$
U_{i}=x_{\text {self }}+\gamma\left[(1-\delta) \min \left\{x_{\text {self }}, x_{\text {other }}\right\}+\delta\left(x_{\text {self }}+x_{\text {other }}\right)\right],
$$

where $\gamma \in(0,1)$ measures how much the participant cares about maximizing the social welfare, and $\delta \in(0,1)$ measures how much the participant cares about maximizing the total social surplus (i.e., $\left(x_{\text {self }}+x_{\text {other }}\right)$ ) versus helping the worse-off person (i.e., $\min \left\{x_{\text {self }}, x_{\text {other }}\right\}$ ). Setting $\gamma=1$ means that the participant cares no more (or less) about their own payoffs than others' payoffs, while setting $\gamma=0$ corresponds to a purely self-interested preference. Setting $\delta=1$ corresponds to a total-surplus maximization while $\delta=0$ means that the social welfare is measured solely according to how well off the least well off is. 
We computed the probability of participants' choices according to the softmax function:

$$
P(\text { option } 1)=\frac{e^{\tau \cdot U(\text { option } 1)}}{e^{\tau \cdot U(\text { option } 1)}+e^{\tau \cdot U(\text { option } 2)}}
$$

Here, $\tau$ is the softmax inverse temperature parameter reflecting sensitivity to differences in utility, where the lower $\tau$ is, the more diffuse and variable the choices are $(\tau>0)$.

All 24 trials and all choice data from five visits per participants were used for model parameter estimation. We used hierarchical Bayesian estimation ${ }^{39,40}$, which assumed each individuals as random samples from a common group-level distribution. For each parameter $(\gamma, \delta$, and $\tau)$, a group-level distribution was set as Gaussian with two hyperparameters - a group-mean and a group-standard deviation - and defined following noncentered parameterization ${ }^{41}$. To constrain the range of parameters, we applied an exponential transformation for the inverse temperature and an inverse probit transformation for the other two weight parameters. All hyperparameters were estimated using uninformative priors $(\operatorname{Normal}(0,10)$ for group-means and Cauchy $(0,2.5)$ with lower bound of zero for group-standard deviations), and we used Markov chain Monte Carlo (MCMC) sampling with the No-U-Turn variant of the Hamiltonian Monte Carlo technique implemented in Stan ${ }^{42}$ and its interface to $\mathrm{R}^{41} .5000$ samples were drawn in each chain and the first 2000 samples were discarded for burn-in. Four chains were run in total and were visually inspected for convergence and good mixing. All values of the potential scale reduction factor (Rhat) for all the variables were less than $1.06^{43}$. To examine individual differences within each parameter, medians were extracted from each individual's parameter distribution.

To assess the identifiability of our model, we conducted a simulation-recovery analysis after the parameters $(\gamma, \delta$, and $\tau)$, were obtained. We fit the model to the simulated data and estimated parameters. The correlations between these parameters and the known generating parameters were then tested with Pearson correlation coefficient. A higher correlation coefficient indicates a more successful parameter recovery.

We also investigated the change in $\gamma$ (i.e., weight on social welfare) and $\delta$ (i.e., weight on total social surplus) over time with repeated measure (T1/T3/T5/T7/T9 in the 2020 cohort; week1/week3/week5 in the 2021 cohort) ANOVA tests.

With linear mixed-effects regressions, in each cohort, we tested the effects of the 7-day change in new cases and the number of new cases per se on $\gamma$ over time respectively, with slope and intercept included for random effects, controlling for data acquisition time, sex, and age $(\gamma \sim 7$ day change in new cases + time + sex + age + (time $\mid$ participant $) ; \gamma \sim$ new case number + time + sex + age $+($ time $\mid$ participant $))$. The same analyses were also done for $\delta(\delta \sim 7$-day change in new cases + time + sex + age $+($ time $\mid$ participant $) ; \delta \sim$ new case number + time + sex + age + (time $\mid$ participant)).

Self-prioritization in the self-tagging task. Self-prioritization was measured by accuracy of self-matched (i.e., the label was self and shape was correctly paired) subtracted by that of stranger-matched (i.e., the label was stranger and shape was correctly paired) pairs. We first investigated the fluctuation of self-prioritization over time with a repeated measure (data 
acquisition time: T1/T3/T5/T7/T9) ANOVA test in the 2020 cohort, and another repeated measure (data acquisition time: week1/week3/week5) ANOVA test in the 2021 cohort.

Further, to examine the association between COVID-19 severity and self-prioritization over time, we used linear mixed-effects regressions. In each cohort, we tested the effects of the 7-day change in new cases and the number of new cases per se on self-prioritization separately, with slope and intercept included for random effects. We controlled for data acquisition time, sex, and age (self-prioritization $\sim 7$-day change in new cases + time + sex + age + (time $\mid$ participant); self-prioritization $\sim$ new case number + time + sex + age $+($ time $\mid$ participant $)$ ).

Association between self-prioritization and altruistic choice. To examine the association between self-prioritization and altruism over time, with linear mixed effect regressions, we tested the following effects with slope and intercept included for random effects, while controlling for time, sex and age:

1) the effect of self-prioritization on altruistic choices over time in each cohort (altruistic choice $\sim$ self-prioritization + time + sex + age $+($ time $\mid$ participant $)$ );

2) the effect of self-prioritization on each of the model-based parameters in DG over time in each cohort $(\gamma \sim$ self-prioritization + time + sex + age $+($ time $\mid$ participant $) ; \delta \sim$ self-prioritization + time + sex + age + (time $\mid$ participant $) ; \tau \sim$ self-prioritization + time + sex + age $+($ time $\mid$ participant));

3) the effect of the change in self-prioritization between adjacent time points on the change in altruistic choices over time in the 2020 cohort (change in altruistic choice $\sim$ change in selfprioritization + time + sex + age $+($ change in self-prioritization + time $\mid$ participant $))$;

4) the effect of the change in self-prioritization between adjacent time points on the change in each of the model-based parameters in DG over time in the 2020 cohort (change in $\gamma \sim$ change in self-prioritization + time + sex + age + (time $\mid$ participant); change in $\delta \sim$ change in selfprioritization + time + sex + age + (time $\mid$ participant); change in $\tau \sim$ change in self-prioritization + time + sex + age $+($ time $\mid$ participant $))$.

Finally, to explore the correlations between self-prioritization and altruism in each cohort, Pearson correlation coefficients were calculated for pairs of self-prioritization in the self-tagging task and the probability of altruistic choices as well as model-based parameters in the DG. Correlations were done for these measures at each time point and for changes in these measures between adjacent time points.

\section{Statistical analyses}

Mixed model regressions were implemented with lme $4{ }^{44}$ and lmerTest ${ }^{45}$ packages in R studio software ${ }^{46}$ (version 1.0.136) and R software ${ }^{47}$ (version 3.3.3). ANOVA tests, $t$ tests, and correlations (Pearson's correlation coefficient; multiple comparisons corrected by BenjaminiHochberg false discovery rate) were implemented using SPSS (IBM SPSS Statistics Version 21.0, IBM Corp.). All $p$ values are 2-tailed. Significance was set at $p<.05$.

\section{Code availability}


The code used to analyze data in the current study is available from the corresponding author on request.

\section{Acknowledgments}

The study was funded by internal funding from the Icahn School of Medicine at Mount Sinai. We thank Jae Shin for developing online versions of the tasks.

\section{Author contributions}

All authors designed the study and participated in data collection. Y.L. A.S. and D.C. analyzed the data. Y.L., A.S., D.C., and X.G. interpreted the data. Y.L., A.S., D.C., and X.G. wrote the manuscript and incorporated edits from all authors.

\section{Data availability}

The datasets generated and/or analyzed during the current study are available from the corresponding author on request.

\section{Competing interests}

The authors declare no conflicts of interest.

\section{References}

1 Henrich, J. et al. Economic man in cross-cultural perspective: behavioral experiments in fifteen small-scale societies. (2001).

2 Nowak, M. A. \& Sigmund, K. Evolution of indirect reciprocity by image scoring. Nature 393, 573-577 (1998).

3 Schwartz, B. Why Altruism Is Impossible... and Ubiquitous. Moral Psychology: Historical and Contemporary Readings, 148 (2010).

4 Fehr, E. \& Gächter, S. Altruistic punishment in humans. Nature 415, 137-140 (2002).

5 Fehr, E. \& Fischbacher, U. The nature of human altruism. Nature 425, 785-791 (2003).

6 De Waal, F. B. Putting the altruism back into altruism: the evolution of empathy. Annu. Rev. Psychol. 59, 279-300 (2008).

7 Bester, H. \& Güth, W. Is altruism evolutionarily stable? Journal of Economic Behavior \& Organization 34, 193-209 (1998).

8 Teoh, Y. Y., Yao, Z., Cunningham, W. A. \& Hutcherson, C. A. Attentional priorities drive effects of time pressure on altruistic choice. Nature communications 11, 1-13 (2020).

9 Eckel, C. C. \& Grossman, P. J. Altruism in anonymous dictator games. Games and economic behavior 16, 181-191 (1996). 
10 Rand, D. G., Greene, J. D. \& Nowak, M. A. Spontaneous giving and calculated greed. Nature 489, 427-430 (2012).

11 Von Dawans, B., Fischbacher, U., Kirschbaum, C., Fehr, E. \& Heinrichs, M. The social dimension of stress reactivity: acute stress increases prosocial behavior in humans. Psychological science 23, 651-660 (2012).

12 Cannon, W. B. Bodily changes in pain, hunger, fear, and rage. (D. Appleton and company, 1915).

13 Del Rey, A., Chrousos, G. \& Besedovsky, H. The hypothalamus-pituitary-adrenal axis. (Elsevier, 2008).

14 Jansen, A. S., Van Nguyen, X., Karpitskiy, V., Mettenleiter, T. C. \& Loewy, A. D. Central command neurons of the sympathetic nervous system: basis of the fight-or-flight response. Science 270, 644-646 (1995).

15 Cooper, D. J. \& Kagel, J. H. Other-regarding preferences. The handbook of experimental economics 2, 217 (2016).

16 Zaki, J. Catastrophe compassion: Understanding and extending prosociality under crisis. Trends in cognitive sciences (2020).

17 Vinkers, C. H. et al. Time-dependent changes in altruistic punishment following stress. Psychoneuroendocrinology 38, 1467-1475 (2013).

18 Klimecki, O. M., Mayer, S. V., Jusyte, A., Scheeff, J. \& Schönenberg, M. Empathy promotes altruistic behavior in economic interactions. Scientific reports 6, 1-5 (2016).

19 Camerer, C. F. Behavioral game theory: Experiments in strategic interaction. (Princeton University Press, 2011).

20 Sui, J., He, X. \& Humphreys, G. W. Perceptual effects of social salience: evidence from self-prioritization effects on perceptual matching. Journal of Experimental Psychology:

Human perception and performance 38, 1105 (2012).

21 Charness, G. \& Rabin, M. Understanding social preferences with simple tests. The Quarterly Journal of Economics 117, 817-869 (2002).

22 Sui, J., Rotshtein, P. \& Humphreys, G. W. Coupling social attention to the self forms a network for personal significance. Proceedings of the National Academy of Sciences 110, 7607-7612 (2013).

23 Moreland, A. et al. Timing of state and territorial COVID-19 stay-at-home orders and changes in population movement-United States, March 1-May 31, 2020. Morbidity and Mortality Weekly Report 69, 1198 (2020).

24 Lemieux, F. The impact of a natural disaster on altruistic behaviour and crime. Disasters 38, 483-499 (2014).

25 Páez, D., Basabe, N., Ubillos, S. \& González-Castro, J. L. Social sharing, participation in demonstrations, emotional climate, and coping with collective violence after the March 11 th Madrid bombings 1. Journal of Social Issues 63, 323-337 (2007).

26 Hartman, A. \& Morse, B. Violence, Empathy, and Altruism: Evidence from the Ivoiran Refugee Crisis in Liberia. British Journal of Political Science 50, 731-755, doi:10.1017/S0007123417000655 (2020).

27 Li, Y., Li, H., Decety, J. \& Lee, K. Experiencing a natural disaster alters children's altruistic giving. Psychological science 24, 1686-1695 (2013).

28 Vollhardt, J. R. Altruism born of suffering and prosocial behavior following adverse life events: A review and conceptualization. Social Justice Research 22, 53-97 (2009). 
29 Staub, E. \& Vollhardt, J. Altruism born of suffering: The roots of caring and helping after victimization and other trauma. American Journal of Orthopsychiatry 78, 267-280 (2008).

30 Lim, D. \& DeSteno, D. Past adversity protects against the numeracy bias in compassion. Emotion (2019).

31 Jordan, J., Yoeli, E. \& Rand, D. Don't get it or don't spread it? Comparing self-interested versus prosocially framed COVID-19 prevention messaging. (2020).

32 Fehr, E. \& Camerer, C. F. Social neuroeconomics: the neural circuitry of social preferences. Trends in cognitive sciences 11, 419-427 (2007).

33 De Quervain, D. J., Fischbacher, U., Treyer, V. \& Schellhammer, M. The neural basis of altruistic punishment. Science 305, 1254 (2004).

34 Brañas-Garza, P. Poverty in dictator games: Awakening solidarity. Journal of Economic Behavior \& Organization 60, 306-320 (2006).

35 Chen, Y. \& Li, S. X. Group identity and social preferences. American Economic Review 99, 431-457 (2009).

36 Dovidio, J. F., Gaertner, S. L., Pearson, A. R. \& Riek, B. M. in Social Identification in Groups (Advances in Group Processes Vol. 22) (eds Shane R. Thye \& Edward J. Lawler) 231-260 (Emerald Group Publishing Limited, 2005).

37 Drury, J. The role of social identity processes in mass emergency behaviour: An integrative review. European Review of Social Psychology 29, 38-81 (2018).

38 Almukhtar, S. et al. in The New York Times (2020).

39 Ahn, W.-Y., Haines, N. \& Zhang, L. Revealing neurocomputational mechanisms of reinforcement learning and decision-making with the hBayesDM package.

Computational Psychiatry 1, 24-57 (2017).

40 Daw, N. D. Trial-by-trial data analysis using computational models. Decision making, affect, and learning: Attention and performance XXIII 23 (2011).

41 RStan: the R interface to Stan v. 2.10.1, 2016 (2017).

42 Carpenter, B. et al. Stan: a probabilistic programming language. Grantee Submission 76, 1-32 (2017).

43 Gelman, A. \& Rubin, D. B. Inference from iterative simulation using multiple sequences. Statistical science 7, 457-472 (1992).

44 Bates, D., Mächler, M., Bolker, B. \& Walker, S. Fitting Linear Mixed-Effects Models Using lme4. 2015 67, 48, doi:10.18637/jss.v067.i01 (2015).

45 Kuznetsova, A., Brockhoff, P. B. \& Christensen, R. H. B. lmerTest Package: Tests in Linear Mixed Effects Models. 2017 82, 26, doi:10.18637/jss.v082.i13 (2017).

46 RStudio: integrated development environment for R (RStudio, PBC., Boston, MA, 2012).

47 R: A language and environment for statistical computing (R Foundation for Statistical Computing, Vienna, Austria, 2010). 


\section{Supplementary Files}

This is a list of supplementary files associated with this preprint. Click to download.

- COVIDALSI.docx 